\title{
Spontaneous Intra-Peritoneal Urinary Bladder Rupture Complicating Benign Prostate Hyperplasia: Case Report
}

\begin{abstract}
Ajape AA ${ }^{1}$ FWACS, Abdulkadir AY ${ }^{2}$ FMCR, Babata AL ${ }^{1}$ FRCS, FWACS, Adesiyun OAM ${ }^{2}$ FWACS(Rad). 1-Division of urology, Department of Surgery and 2-Department of Radiology, University of llorin Teaching Hospital, llorin. Nigeria. Correspondence: Dr. AA Ajape P.0. Box 4850, GP0, Ilorin. Nigeria. E-mail: ajsnrkudimoh@yahoo.co.uk, ajsnrkudimoh@gmail.com Phone: +234-803-574$0982,+234-807-098-4355$
\end{abstract}

\section{Summary}

A case of a rare atraumatic spontaneous rupture of the urinary bladder in a 62-year-old man with benign prostate hyperplasia who presented with anuria, abdominal pain and abdominal distension is reported. He had declined prostatectomy for two and a half years on financial ground. In addition to the presenting history, the presence of free intraperitoneal fluid and abdominal rigidity heightened the clinical suspicion of the diagnosis. This was confirmed by the laboratory demonstration of uroperitoneum and ultrasonographic demonstration of rent in the urinary bladder wall. About 6 litres of urine, mixed with blood, was drained following a moderately difficult trans-urethral bladder catheterization. This led to the spontaneous disappearance of the abdominal distension and healing of the bladder rupture as confirmed by ultrasonography and cystography on the eighth day of presentation. He had urethro-cystoscopy and retropubic prostatectomy electively two months later and has remained well on follow up.

\section{Introduction}

Spontaneous rupture of the urinary bladder is rare and commonly follows bladder wall pathology such as tumour, inflammation, wall weakness from over-distension and outlet obstruction (1-6). The diagnosis may be missed because of non-pathognomonic presentations until at laparotomy, autopsy or during radiological work up (3). Laboratory findings are supportive. Radiological methods such as ultrasonography, contrast cystography and computed tomographic cystography are diagnostic (4). The treatment options include laparoscopic or open surgical repair (7). However, spontaneous healing can be achieved with trans-urethral continuous catheter drainage $(11,12)$, as it was encountered in the case being reported where the uncommon spontaneous intraperitoneal bladder rupture followed an even more rare cause, benign prostate hyperplasia.

\section{Case Report}

A 62-year-old man presented at our Urology unit because of progressive abdominal distension, abdominal pain and anuria of five days duration. He had initially gone into acute urinary retention (AUR) but the painful urge to micturate later abated. This was soon followed by the abdominal distension and discomfort. He neither had history of trauma nor urethral instrumentation. He was not a known peptic ulcer disease patient and no ingestion of non-steroidal anti-inflammatory drug. There was no fever or other constitutional symptoms.

He was a known patient, of the unit, who had histological confirmation of benign prostate hyperplasia two and a half years earlier, but declined prostatectomy on financial ground.

Physical examination showed a middle-aged man in distress. He was tachypnoeic and tachycardic with respiratory rate of 34 cycles per minute and pulse rate of 124 beats per minute respectively. The blood pressure was 170/90mmHg. Abdomen was notably distended, tense and tender with no differential fullness of the suprapubic region. The prostate was clinically enlarged with benign features on rectal examination. The chest was clinically clear. A clinical diagnosis of spontaneous intraperitoneal urinary bladder rupture was made.

Abdomino-pelvic ultrasound scan revealed extensive sonolucent intra-abdominal fluid with the bowel loops freely floating. There was a wide rent of the bladder wall with mucosal to serosal bridging posteriorly leading to free communication between fluids in the urinary blad- 
der and the rectovesical pouch (figure 1). Lack of funds hindered emergency contrast cystography which would have been more objective in delineating the bladder rupture.

Laboratory investigation revealed packed cell volume of $28 \%$, raised serum urea of $26.7 \mathrm{mmol} / \mathrm{L}$, and the serum electrolytes were within normal limits. The peritoneal fluid analysis showed numerous red blood cells and white blood cells per high power field. The urea level of the peritoneal fluid was also high $(25 \mathrm{mmol} / \mathrm{L})$, suggestive of urinary ascites and it was negative for malignant cell.

A Foley urethral catheter (size 20FR) was passed, with slight difficulty. About six litres of urineferous fluid mixed with blood was drained within 30-minutes of catheterization at presentation with subsequent disappearance of the abdominal swelling. Emergency exploratory surgery was hindered by funds and logistics thusperitoneal irrigation was done via the urethral catheter with normal saline. Thereafter, he was maintained on continuous bladder drainage, which led to good bladder healing as confirmed by ultrasonography and contrast cystography, done on the eighth day of admission (figure 2). Patient had urethro-cystoscopy, which revealed an enlarged prostate with intact bladder wall and mucosal trabeculation. He had retropubic prostatectomy two months later and has remained well.

\section{Discussion}

Atraumatic rupture of the urinary bladder is rare and could be life threatening (1-6). Bladder rupture associated with benign prostate hyperplasia is extremely rare in the literature. To the authors' knowledge, we found only two cases of spontaneous urinary bladder rupture, reported by Mellema et al (9) and Benchekroun et al (10), complicating benign prostate hyperplasia. Other cases found associated with benign prostate gland enlargement occurred as complication of therapeutic interventions.

Some of the identifiable predispositions to spontaneous bladder rupture include intrinsic bladder pathology such as tumour, tuberculosis, stones, a diverticulum, a surgical scar, radiotherapy (either directly in the treatment of bladder tumour or in other pelvic tumour) (1-6). In addition, it may follow entero-cystoplasty for small-capacity-high-pressure bladder, eosinophillic cys-

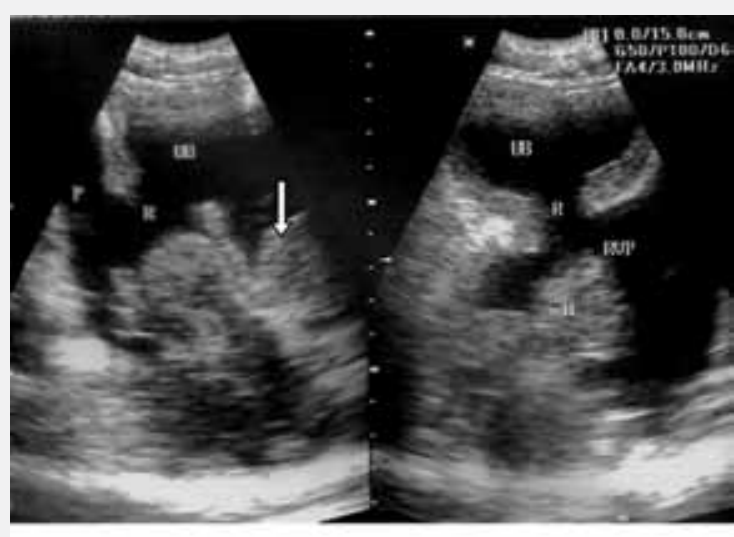

Figure 1A: Sonograms of a 62-year-old man with spontaneous intraperitoneal bladder rupture (longitudinal scan to the left and transverse scan to the right) following benign prostate hyperplasia showing a wide rent $(R)$ in the posterior wall of the urinary bladder (UB), fluid in the retro-vesical pouch (RVP),

haematoma $(H)$, peritoneal fluid $(p)$ and the enlarged prostate (arrow).

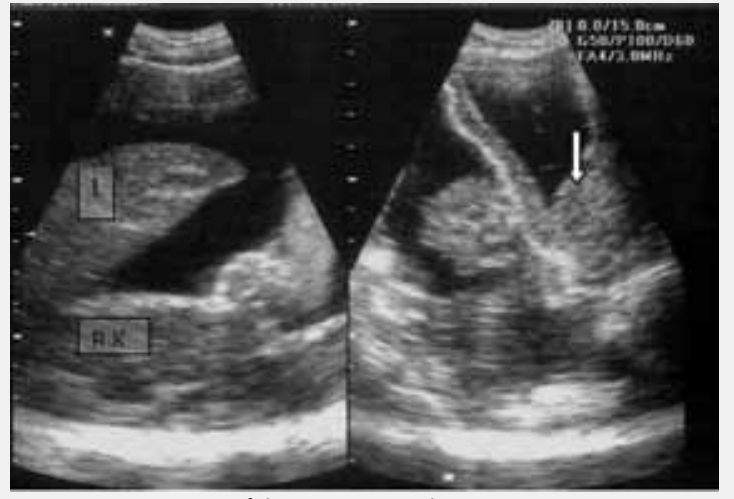

Figure 1B: Sonograms of the same patient demonstrating extensive intraperitoneal fluid around the liver $(L)$ and right kidney $(R K)$ to the left. The right image shows thickened bladder wall, enlarged prostate $(P)$, retrovesical haematoma $(H)$ and pelvic fluid.

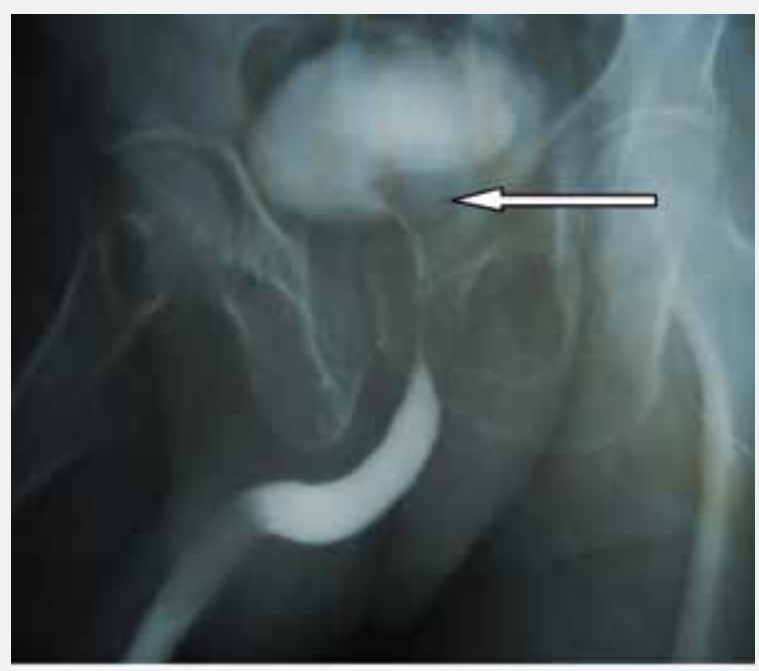

Figure 2: retrograde urethrography done on the 8th day of admission. It shows elevated bladder base and intact bladder wall following spontaneous healing. 
Spontaneous Intra-Peritoneal Urinary Bladder Rupture Complicating Benign Prostate Hyperplasia:

Case Report Ajape AA, Abdulkadir AY, Babata AL, Adesiyun OAM

titis and acute alcohol binge $(1,3,4,6)$. Rarely, it may follow bladder outlet obstruction such as calculus, posterior urethral valve and benign prostate hyperplasia as in this case $(3,9,10)$.

Four groups of aetiological mechanisms leading to bladder rupture was identified by Haddad et al $(1,2)$; these include dull sensorium, weakening of the bladder wall, increased intra-vesical pressure (with or without bladder outlet obstruction) and vascular lesions (radiotherapy, arterial embolism and vesical infarction). The common denominator for these four groups of etiological factors is vesical infarction following ischemia due to over distention of the bladder $(1,2)$. The theory of bladder infarction is unlikely in our patient in view of the spontaneous bladder healing. Tissue necrosis that will delay healing would be expected in bladder rupture due to infarction. The rupture in our patient was most probably the resultant effect of high intra-vesical pressure due to bladder outflow obstruction.

Intraperitoneal bladder rupture is often not suspected leaving the actual diagnosis to be made at autopsy, at surgical exploration or following radiological studies $(1-3,8)$. The delay in diagnosis may result in significant reabsorption of urea and creatinine through the peritoneum causing a significant elevation in blood urea and creatinine levels (8). In such instances, it could be misdiagnosed for renal failure especially if the ascitic fluid is not biochemically analyzed (8). Thus, early diagnosis requires a high degree of clinical suspicion and an increased awareness by the surgeon of the possibility of intraperitoneal bladder rupture $(2,3)$, as demonstrated in this index case report.

According to Haddad et al (2), the diagnosis of spontaneous bladder ruptures are made difficult due to nonspecificity of symptoms. However, affected patients may present with the triad of symptoms of pain, difficulty or inability to void and rigidity of the abdomen (2). These symptoms triad led to the clinical suspicion of bladder rupture in our patient and the subsequent confirmation of diagnosis by both laboratory and radiological evaluation.

Radiology is central to the diagnosis of intraperitoneal bladder rupture. The most valuable diagnostic tool remains cystography $(1,2,4)$. However, because this condition can be life-threatening, ultrasonography or computed tomographic (CT) cystography can suffice $(1,2)$. Emergency ultrasonic evaluation was diagnostic in our patient. Because of its real time advantages, we were able to visualize movement of fluid from intraperitoneum and the bladder via the rent during catheter drainage and saline infusion.

When available, CT scan and Magnetic Resonance Imaging (MRI) will improve the pre-operative diagnostic yields. These modalities were not considered in this patient not only for financial limitation but also for nonavailability at our centre.

The elevation of the blood urea as noted in our patient has been reported as a sensitive indicator of intraperitoneal bladder rupture $(1,2,8)$.

The treatment modality has hitherto been operative management either by open exploration and repair or lately by laparoscopic repair (7). However, many reports have documented the effectiveness of conservative management of spontaneous bladder rupture even in those with recurrent rupture $(11,12)$. The effectiveness of this technique was also proven by this present report with demonstration of spontaneous healing by the eighth day after presentation.

In conclusion, spontaneous rupture of the bladder from benign prostate enlargement is extremely rare. It should be considered, with high index of suspicion, in patient with progressive lower urinary symptoms and a sudden onset of abdominal pain, abdominal swelling and inability to void. Conservative management in form of continuous bladder drainage can suffice in selected stable patient.

\section{References}

1. Haddad FS, Pense S, Christenson S. Spontaneous intraperitoneal rupture of the bladder. J Med Liban. 1994; 42:149-54.

2. Haddad FS, Wachtel TL. Spontaneous intraperitoneal rupture of the bladder. Urol Int. 1987; 42:467-9.

3. Basavaraj DR, Zachariah KK, Feggetter JGW. Acute abdomen: Remember spontaneous perforation of the urinary bladder. J R Coll Surg Edin. 2001; 46:316-7.

4. Pradhan AA. Spontaneous rupture of bladder: A rare clinical entity. MJAFI. 2007; 63 : 92-93

5. Ketata S, Boulaire JL, Al-Ahdab N, et al. Spontaneous intraperitoneal perforation of the bladder: a late complication of radiation therapy for prostate cancer. Clin Genitourin Cancer. 2007; 5:287-90.

6. Mbibu NH, Rafindadi AH, Khalid L. Spontaneous rupture of the bladder due to haemangioendothelioma: A case report. Nig J Surg Res 2001; 3: 100-103. 
7. Gunnarsson U, Heuman R. Intraperitoneal rupture of the urinary bladder: the value of diagnostic laparoscopy and repair. Surg Laparosc Endosc. 1997; 7:53-5.

8. Heyns CF, Rimington PD. Intraperitoneal rupture of the bladder causing the biochemical features of renal failure. $\mathrm{Br} \mathrm{J}$ Urol 60: 217, 1987.

9. Mellema CT, Henneman O, Leenarts JA, Puylaert JB. Spontaneous rupture of the bladder. Ned Tijdschr Geneeskd. 2004; 148:735-40.

10. Benchekroun A, Lachkar A, Soumana A, et al. Spontaneous rupture of bladder diverticulum. Apropos of a case. Ann Urol 1998; 32: 367-9.

11. Leyland JW, Masters JG. Conservative management of an intraperitoneal rupture of an augmentation cystoplasty and continent urinary diversion in an adult. J Urol 2003; 170:524.

12. Slaton JW, Kropp KA. Conservative management of suspected bladder rupture after augmentation entero-cystoplasty. J Urol 1994; 152: 713 